\title{
Propuesta metodológica para el estudio de la presencia del sexo en los cibermedios
}

\section{Methodological proposal for the study of the presence of sex in cybermedia}

Jose-Luis Argiñano - Universidad del País Vasco / Euskal Herriko

Unibertsitatea (UPV/EHU) - joseluis.arguinano@ehu.eus

Rubén Olveira Araujo - Universidad del País Vasco / Euskal Herriko

Unibertsitatea (UPV/EHU) - rubolver@gmail.com

Abstract: Es irrefutable la presencia del sexo en los medios de comunicación. Sin embargo, su representación mediática parece estar limitada por una visión sesgada que encajona el sexo como agente opresor. Del mismo modo, las escasas investigaciones académicas que se han centrado en esta dimensión lo han hecho desde una perspectiva de género, que entiende el sexo como fuente de desigualdades. Teniendo en cuenta que la teoría de la agenda-setting ha demostrado que los medios de comunicación son capaces de moldear el imaginario colectivo mediante la preeminencia de unos temas y encuadres sobre otros, es indudable la influencia de los news media en la manera en la que la sociedad piensa y afronta la cuestión sexual. Con el objetivo de abordar la representación de esta dimensión de una forma holística y no únicamente desde una perspectiva de poder, este trabajo presenta una metodología para análisis de la representación mediática del sexo y, más concretamente, del "sexo que se hace» en los cibermedios. Para ello, se han identificado una serie de nociones que tienden a asociarse a esta dimensión. La clasificación de las informaciones en función de su carácter positivo o negativo, junto a la tipología de los titulares y las temáticas, son otras variables que también se tienen en cuenta y que, paralelamente, contribuyen a identificar características del periodismo de servicio 


\section{Las nuevas narrativas, en el entorno social \\ Universidad de La Laguna, diciembre de 2019}

Keywords: sexo; agenda-setting: periodismo de servicio; metodología; análisis de contenido; cibermedios.

\section{Introducción}

El sexo es una condición que atraviesa la biografía humana incluso desde antes del primer aliento y que no expira hasta el último. Dada su importancia, es irrefutable que tenga su eco en el ámbito público y en los medios de comunicación de masas, entre ellos también en aquellos de carácter más informativo. De hecho, se puede deducir de teorías como la agenda-setting que la representación que se hace del sexo en los news media potencialmente puede empoderar o problematizar la sexualidad de las personas. Si bien existen investigaciones académicas que abordan el hecho sexual humano en los medios de comunicación, estas lo han hecho desde una perspectiva de género, que entiende el sexo como fuente de desigualdades. Sin embargo, no se ha hallado ningún acercamiento desde un enfoque más holístico. Este trabajo presenta una propuesta metodológica para el análisis del sexo en los medios de comunicación online desde una perspectiva integral, con un enfoque desde la sociología de la información.

\section{Marco teórico}

Antes de continuar, cabe destacar que cuando hablamos de sexo no siempre hablamos del mismo sexo. Esta potencial confusión se debe, entre otros motivos, a que este se ha ido conformando epistemológicamente a través de diferentes nociones, pero también a la fusión intelectual de dimensiones diferentes y diferenciables mediante el uso compartido de la raíz semántica sex (Landarroitajauregi, 2013). Se pueden distinguir al menos tres.

La primera hace referencia al sexo como condición sexual o «sexo que se es». De hecho, el significado original del lexema sex proviene del participio latino sexare, que se puede traducir como cortado, seccionado, separado; es decir, diferenciado. Por tanto, hace alusión a la condición de ser diferentes $-\mathrm{y}$ únicos- por razón de sexo. Sin embargo, en el imaginario actual la raíz semántica sex también se relaciona, por un lado, con la conducta sexual o 


\section{Las nuevas narrativas, en el entorno social}

\section{Universidad de La Laguna, diciembre de 2019}

«sexo que se hace» y, por otro, con atributos sexuales o «sexo que se tiene». Esta segunda forma de entender y acotar el sexo a las prácticas -y en menor medida, a los deseos- fue potenciada por la segunda generación de sexólogos con William Masters y Virginia Johnson a la cabeza, mientras que la tercera, si bien lleva siglos presente, fue reforzada y extendida por la popular distinción entre sexo y género del endocrinólogo John Money.

Desde el enfoque sexológico se propone una definición integradora del sexo, siendo este aquella «condición multifactorial -presente al menos en los dominios biológico, psicológico y cultural- que especializa y diferencia intersexualmente y clasifica dimórficamente a los individuos de la mayoría de las especies vivas, propiciando una función que llamamos anhelo del encuentro y que, potencialmente, procura sinergia» (Landarroitajauregi, 2013). Es decir, se jerarquiza la condición del «sexo que se es» sobre la conducta del «sexo que se hace» y los atributos del «sexo que se tiene», pues la capacidad explicativa de esta integra las dos siguientes, mientras que no ocurre lo mismo a la inversa.

Desde esta panorámica holística del sexo, Landarroitajauregi distingue ocho nociones o unidades cognitivas de significado epistemológico que han trenzado históricamente la forma de entender esta condición. Por orden de aparición, la primera sería procreación o sexo como agente procreador. Le siguen la hedonía o sexo como placer y la erótica o sexo como deseo del otro. A continuación está la sepsis o sexo como mácula -ya sea como pecado, patógeno o delito-. A mediados del siglo XVIII aparece la noción ilustrada del sexo o sexo como diferencia. Posteriormente, con Freud llega el sexo como represión interna y con los movimientos feministas de la segunda mitad del siglo XX el sexo como opresión externa. Finalmente, la corriente sustantiva de la Sexología comprende el sexo como sinergia.

Estas ocho nociones se pueden clasificar en dos grandes grupos: por un lado, las que tratan de responder a la pregunta qué es el sexo y, por otro, las que buscan responder a cómo debería ser el sexo -si bien por su condición reactiva se formulan en negativo: qué no debería ser el sexo-. En el primer grupo estarían cinco nociones: el sexo como procreación, hedonía, erótica, 


\section{Las nuevas narrativas, en el entorno social}

Universidad de La Laguna, diciembre de 2019

diferencia y sinergia, mientras que en el segundo se encuadran las tres nociones restantes: el sexo como sepsis, represión y opresión.

Cabe destacar que estas ocho nociones no solo coexisten a día de hoy en el imaginario posmoderno, sino que se entremezclan unas con otras. Por poner un ejemplo, la erótica o deseo del otro puede convertirse en un tipo de opresión -el denominado amor romántico-, pero también la opresión puede volverse deseo de dominación o sumisión a una persona concreta -como dos de los tipos de anhelos y placeres contemplados dentro de las siglas BDSM-.

Teniendo en cuenta que la actuación humana depende de su interpretación del entorno y que la realidad se construye a través de los significados generados en un proceso de interacción social (Blumer, 1982), la concepción del sexo y sus diferencias -además de los deseos, prácticas y otros resultantes que de él emanan- influyen no solo en los encuentros eróticos, sino también en la interacción social entre los sexos y en la propia experiencia de vivirse uno mismo como ser sexuado.

Tanto la comunicación en general como la agenda mediática en particular juegan un papel crucial en este proceso de construcción de la realidad. De hecho, la teoría de la agenda-setting ha demostrado que los medios de comunicación informativos son capaces de moldear el imaginario colectivo a largo plazo mediante la preeminencia de unos temas y encuadres sobre otros (McCombs, 2006). De esto se puede deducir que la representación del sexo en los news media potencialmente puede empoderar o problematizar la sexualidad de las personas.

La teoría de la agenda-setting distingue entre dos grandes niveles. El primero se refiere a los temas. Aunque la migración desde la prensa de papel a los cibermedios ha reducido el corsé que supone la limitación de espacio de la prensa offline y ha permitido expandir el campo de trabajo de los periodistas, la capacidad de los medios para ofrecer noticias sigue siendo limitada; por lo tanto, deben optar. Respecto a esta preeminencia de unos temas sobre otros podría establecerse una similitud con el coste de oportunidad, que supone decidirse por una inversión en tanto que se renuncia a otra actuación: si se invierte en una estación de trenes se renuncia a un hospital, por ejemplo. En 


\section{Las nuevas narrativas, en el entorno social \\ Universidad de La Laguna, diciembre de 2019}

ambos casos, no es fácil percatarse de la ausencia. De ahí la primera pregunta de investigación en relación al sexo:

Q1. ¿Es el sexo parte de la agenda-setting de los cibermedios?

Pese a compartir ciertas limitaciones, también cabe mencionar que si el proceso de producción de información en un medio tradicional necesita recorrer un amplio camino hasta su emisión, en los cibermedios los tiempos de producción son breves y los condicionantes menores (Iglesias, 2012). Por tanto, los resultados probablemente no son extrapolables a otros soportes, como el papel. Asimismo, hay que incidir en el poder de los medios en este primer nivel se apoya en la tesis de que su influencia es de carácter cognitivo, en lugar de persuasivo.

Por otra parte, si el primer nivel de la agenda-setting indica sobre qué hay que pensar, el segundo postula que la importancia radica no solo en los temas, sino en la forma presentarlos: señalan qué y cómo pensar sobre aquello que previamente se ha indicado como digno de reflexión. Es decir, la forma en que se presenta el tema revierte en la manera en la que la sociedad concibe; en este caso, su sexualidad. Lo que nos lleva a la segunda pregunta de investigación:

Q2. Cuando aparece, ¿qué atributos se le confieren al sexo?

A la hora de decidir qué temas se van a publicar y qué atributos se van a incluir, los periodistas se guían por criterios de noticiabilidad (Armentia, Caminos, 2009). El impacto, el conflicto y la pugna entre las partes son algunos de los principales sesgos informativos, lo cual probablemente conduzca más a nociones del sexo como opresión y represión que como hedonía y erótica. De todas formas, los criterios de noticiabilidad son solamente parte del proceso multifactorial de la construcción de la información. Conviene en este punto remitirse a la sociología del periodismo (Park, 1969; Shoemaker y Reese, 1991), según la cual el trabajo periodístico, la agenda-building, es el resultado del equilibrio entre fuerzas interconectadas como las rutinas laborales, el nivel empresarial, el entorno social y la ideología imperante: «Se trata de concebir las noticias como producto cultural construido colectivamente y de manera diferenciada por distintos actores sociales y factores estructurales y 


\section{Las nuevas narrativas, en el entorno social \\ Universidad de La Laguna, diciembre de 2019}

situacionales que influyen en la definición del contenido noticioso» (Cervantes, 1996;53). Las creencias religiosas o la perspectiva sobre sexo son parte de ese constructo que mediatizan todos y cada uno de los pasos de la construcción de las noticias.

\section{Estado de la cuestión}

Pese a la condición central del sexo en las biografías humanas, prácticamente la totalidad de las investigaciones previas sobre la cobertura mediática del «Sexo que se hace» están realizadas desde una perspectiva de género; es decir, desde un prisma de relaciones de poder en la que la mujer aparece subordinada al hombre. No es de extrañar, por tanto, que esta literatura se haya centrado en la cosificación de la mujer, por un lado, y en las agresiones adjetivadas sexuales, por otro.

Dentro de este primer bloque destacan las investigaciones sobre la cobertura de la prostitución y, especialmente, de la trata (Sobel, 2016; Sobel, Friedman y Johnston, 2017; Suppiah, Kaur, y Shanthi, 2019, etc.). En el segundo, se centran en la representación de las violaciones y los asaltos "sexuales" (Baum, Cohen y Zhukov, 2018; Biressi, 2019; etc.) y, en menor medida, también en los abusos a menores (Christensen, 2017; Gjika, 2019; etc.). Relacionadas con estas dos grandes temáticas, existen investigaciones esporádicas sobre otros asuntos, si bien siempre desde un prisma negativo. Un ejemplo serían los estudios sobre la cobertura de escándalos sexuales (Juntunen y Väliverronen, 2010; etc.).

En cuanto a las revistas dirigidas a un público femenino y a un público adolescente, Ward (2003) señala que las investigaciones previas han identificado reiteradas veces que «deseo sexual» y «riesgo sexual» son los dos enfoques más populares de las noticias relacionadas con la sexualidad. No obstante, nuevamente se ha profundizado considerablemente más en lo segundo que en lo primero. Por ejemplo, los trabajos Durham (1998) y Joshi, Peter y Valkenburg (2011), concluyen que la sexualidad masculina no se encuentra problematizada y se entiende como algo natural y, habitualmente, agresiva; mientras la sexualidad femenina se representa como fuente de 
innumerables calamidades que van desde «riesgos» de embarazo e Infecciones de Transmisión Genital -más conocidas como Infecciones de Transmisión Sexual (ITS)- a presiones, ataques, abusos, acosos, violaciones, etc.

Especialmente destacable es el artículo de Bachechi y Hall (2015), quienes utilizaron como rejilla observacional los discursos tradicionales de pureza, descontento asumido y piedad para analizar el contenido de las revistas dirigidas a un público femenino. Los autores entienden como pureza aquel discurso que transmite una noción «inocente y pura de las mujeres jóvenes y de las niñas»; o dicho de otro modo, sexo como sepsis. El descontento asumido representa a las mujeres "sexualmente activas" como mujeres dañadas por dicha decisión y se vincula a ataques, asaltos, violaciones, embarazos no deseados, prácticas previamente no planificadas que se realizan por presión del hombre, etc.; desde un enfoque sexológico, sexo como opresión. Por último, partiendo de que los dos anteriores discursos entienden la sexualidad femenina como algo de lo que las mujeres deberían protegerse, con piedad se presenta a las mujeres que permanecen en celibato como «satisfechas y con el control sus vidas»; sexo como represión. El nexo de unión entre estos tres discursos es la comprensión de «la actividad sexual» -por el contexto, se puede entender como prácticas penetrativas, presuntamente intravaginales- como algo peligroso para la mujer y, por ende, un apoyo a la castidad femenina, a veces de forma implícita y en otras ocasiones más explícita.

\section{Propuesta metodológica}

Con el objetivo de abordar la representación mediática del «sexo que se hace» de una forma holística, a continuación se ofrecen pautas para llevar a cabo un análisis del contenido, definido como «una técnica de investigación destinada a formular, a partir de ciertos datos, inferencias reproducibles y válidas que puedan aplicarse a su contexto» (Krippendorff, 1990: 28).

\subsection{Nociones del sexo}




\section{Las nuevas narrativas, en el entorno social Universidad de La Laguna, diciembre de 2019}

Como ya se ha mencionado anteriormente, la raíz semántica sex y sobre todo el adjetivo sexual se utilizan para referirse a diferentes dimensiones del sexo; a saber, condición sexual o "sexo que se es», conducta sexual o «sexo que se hace» y atributos sexuales o «sexo que se tiene». Por tanto, aunque el objeto de estudio de esta investigación sea la faceta erótica y hedónica -«sexo que se hace»-, en un primer momento se propone clasificar el corpus en base a estas tres grandes dimensiones, no solo para cribar aquellos artículos que no tengan que ver con el objeto de estudio - «sexo que se es» y «sexo que se tiene»-, sino también para averiguar qué porción ocupan dentro de los usos del lexema sex.

Para continuar con la codificación, los artículos que específicamente mencionen o discurran sobre el «sexo que se hace» se pueden clasificar en función de las siguientes cuatro nociones: sexo como procreación; sexo como valor (subdivido en dos: erótica o deseo del otro; y hedonía o placer); sexo como producto mercantil; y sexo como riesgo (subdividido a su vez en tres: sexo como represión interna; sexo como opresión externa; y sexo como sepsis) (ver tabla 1).

Parte de las nociones ideadas en esta propuesta metodológica para el análisis del «sexo que se hace» son coincidentes con las nociones del sexo de Landarroitajauregi (2013), si bien se han añadido otras, dado que los objetos de estudio difieren: en tanto que las nociones concebidas por este autor están pensadas desde «sexo que se es», este trabajo presta una atención preferente al «sexo que se hace».

Al igual que las nociones propuestas por Landarroitajauregi para «sexo que se es», estas nociones no solo coexisten en el imaginario posmoderno, sino que se entremezclan unas con otras. Teniendo esto en cuenta, se propone establecer dos niveles en la codificación, uno principal y otro secundario, con el fin de establecer jerarquías en dichas interrelaciones. Ambos niveles se nutren de las mismas cuatro nociones de «sexo que se hace», pero la doble codificación para cada pieza informativa aporta mayor concreción y permite conocer mejor la preeminencia de cada una de las nociones. 


\section{Las nuevas narrativas, en el entorno social Universidad de La Laguna, diciembre de 2019}

Asimismo, partiendo de la fuerza que ha tomado la máxima «lo personal es político», también se propone añadir de forma complementaria una distinción sobre la dimensión privada -o intimidad- y la gestión pública del «sexo que se hace».

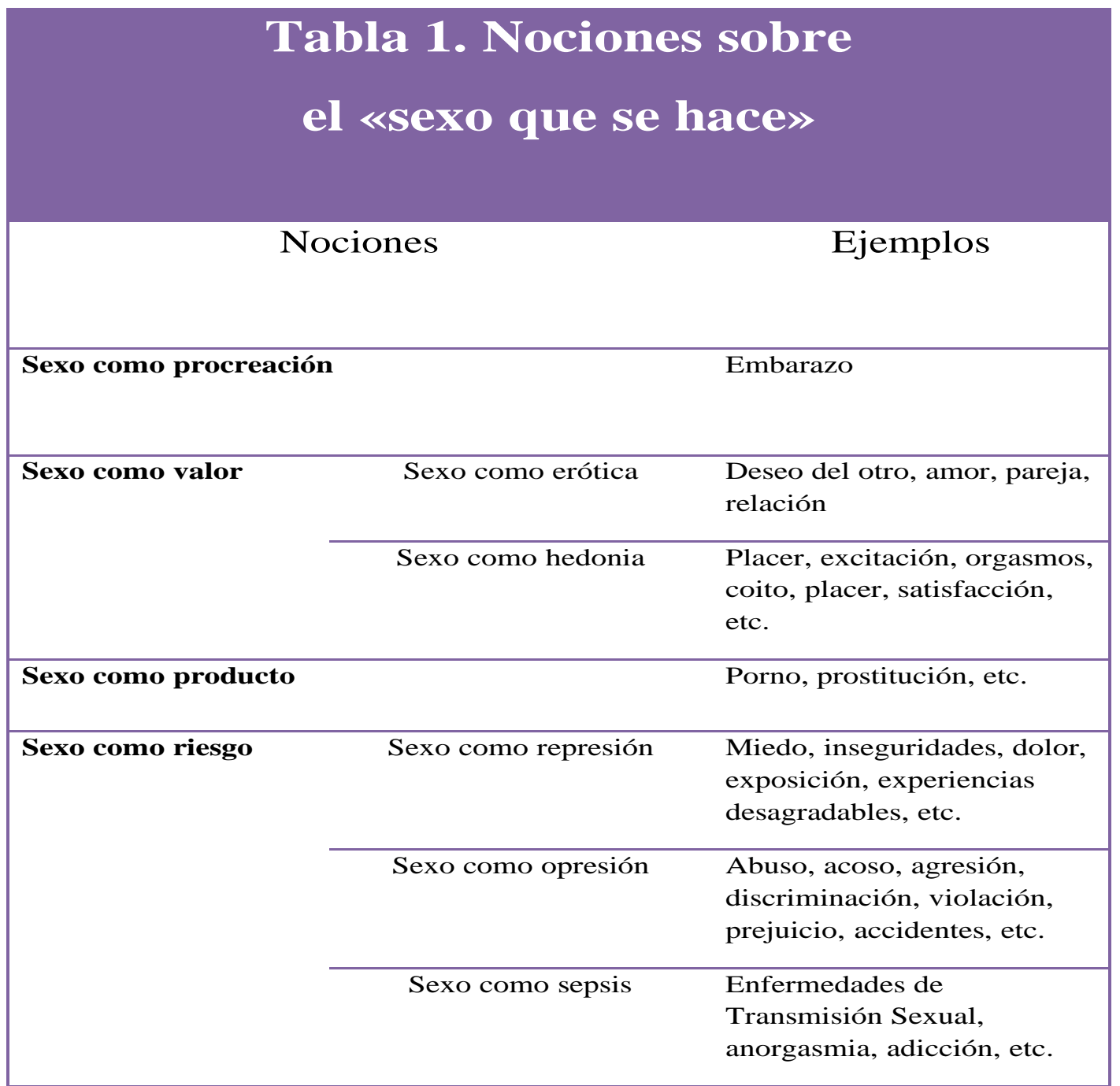

\subsection{Temáticas y tono}

Si se acepta que «la intuición de que el conocimiento tiene que ser estructurado en bloques» (Van Dijk y Kintsch, 1983: 47) es evidente la pertinencia de estructurar el corpus en temas y tonos. El tema, como concepto originario del análisis del discurso, permite delimitar el campo de significado 


\section{Las nuevas narrativas, en el entorno social \\ Universidad de La Laguna, diciembre de 2019}

que da coherencia a la información. Para la investigación de la presencia del sexo en los cibermedios se han inducido cinco categorías temáticas diferentes: política, sociedad, economía, cultura y deportes. Se agrega una sexta división (otros) para las informaciones imposibles de encuadrar en alguna de las categorías definidas.

Al igual que con las nociones de sexo, se plantean dos niveles temáticos: principal y secundario, para dar una mejor solución al carácter trasversal de las informaciones. Por ejemplo, una información sobre prostitución es susceptible de ser encuadrada en economía, política o sociedad. La preeminencia que el cibermedio conceda en su información a cada área permitirá codificar las dos más presentes, y solo una de ellas se considerará hegemónica.

El tono, entendido como carácter evaluativo de la información, es un concepto que ha sido aplicado en otras investigaciones (Henry y Leone 2010; Igartua et al. 2007). Se diferencian el tono positivo, negativo o neutro. Se estima que la información contiene un cariz positivo cuando en la misma se subrayan los beneficios que, en este caso, tiene el «sexo que se hace» (ventajas para la salud, la autoestima, las relaciones sociales, etc.). El cariz negativo, por el contrario, deviene de una información en la que se remarcan las desventajas y/o perjuicios que generan dichas prácticas y deseos. Por último, el tono neutro se relaciona con las piezas periodísticas que se limitan a la divulgación y que adoptan un carácter aséptico. Para ello se toman en consideración las expresiones, citas y palabras que reflejen una valoración sobre el lexema sex.

A la identificación de tema y tono se suma la cuantificación y clasificación de las fuentes informativas utilizadas por los periodistas para construir los diferentes discursos sobre el sexo. Se plantea cotejarlas con los temas y tonos ya fijados para ver sus implicaciones y posibles influencias.

\subsection{Tipos de titulares y periodismo de servicio}

Otra de las variables sistematizadas en esta investigación es el estilo lingüístico a partir de la clasificación de los titulares que encabezan las informaciones seleccionadas. Se han distinguido nueve tipos: informativo, interrogativo, 
expresivo, imperativo, demostrativo, segunda persona, numérico, de citas y apelativo.

Los titulares informativos evitan la carga interpretativa y se limitan a citar el acontecimiento (qué ha hecho quien). Los interrogativos plantean cuestiones que se responden en el texto desde una vocación de periodismo de servicio. Los expresivos apenas aportan información y expresan un estado de ánimo. Se parte de la presunción de que el lector es conocedor de la información. Los encabezamientos imperativos no se limitan a sugerir, sino que incluyen órdenes a los lectores.

Tabla 2. TIPOS DE TITULARES

\section{Informativo \\ Interrogativo}

Expresivo

Imperativo

Demostrativo

Segunda persona

Numérico (listas)

De citas

\section{Apelativo}

Los demostrativos pueden comenzar precisamente con un pronombre demostrativo o sugerirlo de manera implícita y ocultan gran parte de la información para incentivar el interés del lector por el contenido íntegro de la información (esta técnica que también se observa en los titulares con el recurso a la segunda persona). Las listas (titulares numéricos) como forma de titular ofrecen una información más sistematizada (Diezhandino, 1994). Las citas, por otra parte, en el titular suele ser un recurso para historias personales, que sirven de ejemplificación.

Por último, se computan también de forma expresa los titulares apelativos, aunque cabe la posibilidad de que en algunos de los tipos anteriores, salvo en los informativos, se encuentren rasgos definitorios de la apelación. El 


\section{Las nuevas narrativas, en el entorno social}

Universidad de La Laguna, diciembre de 2019

académico Núñez Ladevéze acuñó la siguiente definición para el titular apelativo: «Utiliza el lenguaje para llamar la atención sobre un hecho cuyo conocimiento no se presume, pero del que no se informa» (1991, p. 222). La intención última del periodista es persuadir al lector para que modifique su actitud. Tienen carga expresiva pero también incluyen parte de la información y buscan que el lector se identifique con el medio.

Esta distinción en base a la tipología de los titulares permite abordar de forma sistemática otra variable: el periodismo de servicio. Después de todo, los titulares de segundas personas, los demostrativos o las listas son tres de las tipologías que de forma habitual responden a las características del periodismo de servicio. De esta manera, se puede analizar de forma sistemática y eficiente si los medios ofrecen al lector información práctica, en este caso sobre el «sexo que se hace». En esta tesitura «cobra sentido el concepto de información movilizadora, que empuja al lector a actuar» (Diezhandino, 1994; 100).

Teniendo en cuenta todo lo anterior, la elaboración de una ficha de análisis se antoja imprescindible para codificar no solo aquellas variables ya definidas, sino aquellas también de forma e identificación, como el cibermedio, la fecha, el enlace, la sección, el género, la autoría o el número de comentarios. Esta labor conlleva la generación de un libro de códigos y la validación de los consiguientes resultados mediante el coeficiente de fiabilidad de Holsti (1969).

\section{Discusión}

La escasa literatura académica que se ha dedicado a analizar la representación mediática del «sexo que se hace» lo ha hecho desde una perspectiva de género, con el consiguiente sesgo al entender el sexo como fuente de desigualdades. Este trabajo plantea una propuesta metodológica para un primer abordaje de este objeto de estudio de una forma holística y, de esta manera, sentar las bases para posteriores acercamientos más específicos. Asimismo, la conceptualización del sexo en tres dimensiones diferenciables y la identificación ad hoc de una serie de nociones o unidades cognitivas de significado epistemológico que tienden a asociarse al «sexo que se hace», junto con otras variables propias del análisis de los mensajes mediáticos, 


\section{Las nuevas narrativas, en el entorno social Universidad de La Laguna, diciembre de 2019}

potencialmente convierten esta propuesta en una útil herramienta de análisis; más aún, si se tienen en cuenta los diferentes niveles y sus posibles combinaciones.

Resulta pertinente apuntar que la teoría de la agenda setting, en concreto, su segundo nivel, plantea la correlación existente entre el énfasis que la prensa concede a ciertos atributos y la percepción que de estos asumen los ciudadanos. Es innegable el carácter conflictivo que de forma habitual genera cualquier hecho relacionado con el sexto y, por lo tanto, parece necesario llevar a cabo una labor de investigación que identifiquen el espacio y el rol que los mass-media conceden a las actividades relacionadas con el sexo y, de manera explícita, con aquellas que tienen relación con una perspectiva placentera de la actividad sexual como forma de desarrollo íntegro del ser humano.

\section{Referencias bibliográficas}

Armentia, J. I. y Caminos, J. M. (2009). Redacción Informativa en Prensa. Ariel, Barcelona.

Bachechi, K. N. y Hall, M. (2015). "Purity, presumed displeasure and piety in the 'big three': a critical analysis of magazine discourse on Young women's seuxality", Journal of Gender Studies, 24, 549-560.

Baum, M. A., Cohen, D. K. y Zhukov, Y. M. (2018). "Does Rape Culture Predict Rape? Evidence from US Newspapers, 2000-2013". Quarterly Journal of Political Science, 13, 263-289

Biressi, A. (2019). "Following the money: News, sexual assault and the economic logic of the gendered public sphere". European Journal of Cultural Studies, 22 (5-6), 595-612.

Blumer, H. (1982). El interaccionismo simbólico. Barcelona. Hora.

Cervantes, C. (1996). "Construcción primaria del acontecer y planeación de la cobertura informativa: propuesta metodológica para su estudio". Comunicación y Sociedad, 28, 49-82. 


\section{Las nuevas narrativas, en el entorno social Universidad de La Laguna, diciembre de 2019}

Christensen, L. S. (2017). "The new portrayal of female child sexual offenders in the print media: A qualitative content analysis". Sexuality \& Culture, 22 (1), 176189.

Diezhandino, M. P. (1994). Periodismo de servicio. Barcelona. Bosch.

Durham, M. G. (1998). "Dilemmas of desire: Representations of Adolescent Sexuality in Two Teen Magazines", Youth \& Society, 29, 369-389

Gjika, A. (2019). « New media, old paradigms: News representations of technology in adolescent sexual assault ». Crime, Media, Culture, 1-16.

Henry, E. y Leone, A. (2010). "Measuring qualitive information in capital markets research" (working paper).

\section{https://papers.ssrn.com/sol3/papers.cfm?abstract id=1470807}

Holsti, O. (1969). Content analysis for the social sciences and humanities. Reading, MA, Addison-Wesley.

Igartua, J. J. ; Muñiz, C. ; Otero, J. A.; y De la fuente, M. (2007): "El tratamiento informativo de la inmigración en los medios de comunicación españoles. Un análisis de contenido desde la Teoría del Framing". Estudios sobre Mensaje Periodístico, 13, 91-110.

Iglesias, M. (2012). Rutinas productivas de un cibermedio nativo digital.

Cuadernos de Información, 30, 9-20.

https://www.redalyc.org/articulo.oa?id=971/97124309002

Joshi, S. P., Peter, J. y Valkenburg, P. M. (2011). "Sripts of sexual desire and danger in US and Dutch teen girl magazines: a cross-national content analysis", Sex Roles, 64, 463-474.

Juntunen, L., y Väliverronen, E. (2010). "Politics of sexting: Re-negotiating the boundaries of private and public in political journalism". Journalism Studies, 11(6), 817-831.

Krippendorf, K. (1990). Metodología de análisis de contenido. Teoría y Práctica. Barcelona, Paidós. 


\section{Las nuevas narrativas, en el entorno social Universidad de La Laguna, diciembre de 2019}

Landarroitajauregi, J. (2013). Reflexiones Cítricas para Sexólogos Avezados.

Valladolid. Isesus.

McCombs, M. y Evatt, D. (1995). "Los temas y los aspectos. Explorando una nueva dimensión de la agenda-setting". Comunicación y Sociedad, 8 (1), 7-32.

McCombs, M. y Shaw, D. L. (1972). "The agenda-setting function of the Mass Media”. Public Opinion Quarterly, 36, 176-187.

Núñez-Ladevéze, L. (1995). Introduccion al periodismo escrito. Barcelona. Ariel Park, R. E. (1969) "News as a form of knowledge: a chapter in the sociology of knowledge". American Journal of Sociology, 45, 669-686

Shoemaker, P. y Reese, S. (1991). Mediating the message in the 21st century; a media sociology perspective. Londres. Routledge.

Sobel, M., Friedman, B., y Johnston, A. (2017). "Sex trafficking as a news story: Evolving structure and reporting strategies". Journal of human trafficking, 5 (1), 43-59

Sobel, M. (2016). "Sex trafficking in Thai media: A content analysis of issue framing". International Journal of Communication, 10, 6126-6147

Suppiah, P. C., Kaur, S., Arumugam, N., y Shanthi, A. (2019). "News Coverage of Foreign Sex Workers in Malaysia: A Critical Analysis". Online Journal of Language Studies, 19(1). 136-152

Van Dijk, T. A. y Kintsch, W. (1983). Strategies of discourse comprehension.

New Cork. Academia Press.

Ward, L. M. (2003). "Understanding the role of entertainment media in the sexual socialization of American youth: A review of empirical research", Develomental Reviews, 23, 347-388. 